\title{
Management of Chemotherapy-Induced Neutropenia: Measuring Quality, Cost, and Value
}

\author{
Michaela A. Dinan, PhD ${ }^{a, b}$; Bradford R. Hirsch, MD, MBA ${ }^{\mathrm{a}, \mathrm{b}}$; and Gary H. Lyman, MD, MPH秀
}

\begin{abstract}
Treatment-associated neutropenia continues to represent the most common dose-limiting toxicity of cancer chemotherapy. It often leads to fever and infection, prompting hospitalization and occasionally resulting in serious morbidity, and even mortality, despite modern broad-spectrum antibiotic treatment and supportive care. Neutropenia and its complications may also lead to chemotherapy dose reductions, treatment delays, or early treatment termination, compromising disease control and the potential for cure. NCCN Clinical Practice Guidelines in Oncology recommend administration of primary prophylaxis with a myeloid growth factor in patients receiving regimens associated with a high risk for febrile neutropenia, and consideration of prophylaxis in patients receiving lower-risk regimens who have other risk factors that might place them at higher risk for febrile neutropenia. Although these agents have been shown to be effective and safe in numerous randomized controlled trials, they are expensive and contribute significantly to increasing health care costs. Regulatory agencies and guideline organizations do not currently address the issue of cost. However, with the relentless increase in health care use and current efforts to reform health care, it has become increasingly important to assess both the cost and the net benefit of interventions related to an episode of care in order to compare the overall value of therapeutic options. This article defines and discusses the intersection of quality, costs, and value in the context of prophylactic myeloid growth factor use in patients with cancer receiving myelosuppressive chemotherapy. (J Natl Compr Canc Netw 2015;13:e1-e7)
\end{abstract}

\footnotetext{
From a Duke Cancer Institute and buke Clinical Research Institute, Durham, North Carolina; 'Hutchinson Institute for Cancer Outcomes Research, Fred Hutchinson Cancer Research Center; and dUniversity of Washington Schools of Medicine, Public Health and Pharmacy, Seattle, Washington.

Submitted July 28, 2014; accepted for publication August 10, 2014. Drs. Dinan and Hirsch have disclosed that they have no financial interests, arrangements, affiliations, or commercial interests with the manufacturers of any products discussed in this article or their competitors. Dr. Lyman has disclosed that he is a Principal Investigator on a research grant to the Fred Hutchinson Cancer Research Center from Amgen Inc.

Correspondence: Gary H. Lyman, MD, MPH, Hutchinson Institute for Cancer Outcomes Research, Fred Hutchinson Cancer Research Center and the University of Washington Schools of Medicine, Public Health and Pharmacy, 1100 Fairview Avenue N, M3-B232 Seattle, WA 98109. E-mail: glyman@fredhutch.org
}

\section{Value in Cancer Care}

The cost of cancer care in the United States has doubled over the past decade ${ }^{1}$ and continues to outpace increases in overall health care spending and the national gross domestic product. ${ }^{2}$ An aging population, the increasing use of existing resources, and an uptake of emerging technologies stand to compound the current problem. ${ }^{3}$ Absent the willingness to control the individual costs of drugs, technologies, and reimbursement rates for episodes of care, controlling the use of existing and emerging technologies represents the primary means of mitigating increasing costs. A significant portion of current overall health care expenditures, perhaps as much as $30 \%$, is thought to provide little benefit. ${ }^{4}$ Further evidence of the malleability of cancer care costs is apparent from the wide variability in costs between countries, ${ }^{5}$ and even regionally within the United States. ${ }^{6}$ Despite rapid and large increases in spending, only modest gains in survival have been observed. ${ }^{2}$ As a result, the concept of value in medical decision-making, particularly in oncology, has become a centerpiece of the current health policy discussion. Despite calls for greater awareness of the relative value of diagnostic and therapeutic interventions in medical decision-making and health care policy formulation, the definition of value continues to be a source of confusion. ${ }^{7}$

\section{Definition of Value}

One of the more basic definitions of value-outcomes divided by $\operatorname{costs}^{8}$ — provides a reasonable starting point. However, which outcomes are relevant depends on who is defining them-patients, physicians, payers, policy makers, drug makers, or society — and may include several metrics, such as duration of life, quality of life, health status, equity, adverse outcomes, compassion, and the opportunity to receive care. ${ }^{2,7}$ An alternative definition of value assesses quality divided by costs, arguing that 
Dinan et al

it is not simply quantitative outcomes that are relevant. Agreeing on a definition of quality, however, is equally problematic. Even the definition of costs is unclear, because one must decide which costs are relevant and from what perspective to analyze them. The goals of balancing quality and costs often seem to conflict depending on which stakeholder is being asked. For example, the objective of payers is often to control costs through limiting access to scarce or expensive resources, which can be in direct opposition to the interests of physicians and patients.

Potential metrics of quality depend on the stakeholder and must consider net benefit and harm, and can include access to services, longevity, safety, convenience, patient-centeredness, patient satisfaction, or quality of life, as well as profitability and cost containment. ${ }^{7}$ Longevity is important but does not encompass important issues related to quality of life, including symptoms of disease, comorbidities, and treatment-related toxicity, yet the alleviation of all of these phenomena is of inherent value to patients with cancer and their families. Nevertheless, the perception of quality, including quality of life, varies greatly, and its measurement is fraught with challenges. Other outcome measures report survival time after adjusting for quality of life, as illustrated by the Q-TWIST (Quality-Adjusted Time Without Symptoms or Toxicity), which represents the quality of time without symptoms of disease or toxicity of treatment.

Accounting for costs can be equally challenging and depends on the perspective taken. Costs can include direct medical expenditures, work lost, and caretaker burden, and vary from the perspective of the patient, provider, health system, payer, and society. The results are influenced by sociotemporal, geographic, ethnic, cultural, and health system factors. Even the assessment of direct costs is not straightforward and may vary depending on the region or country being studied. ${ }^{9}$ Expenditures are often of even greater interest, composed of the unit cost and the frequency of service delivery across the broad context of an illness or encounter. Likewise, when measuring costs of care, it is necessary to capture the total costs associated with a condition over its entire course.

Ultimately, the concepts of quality, costs, and value of health care are inescapably linked, with each having an unquestioned impact on the other.
Simply reducing the costs of an intervention without full consideration of the impact on outcomes can create an illusion of progress while limiting effective care. ${ }^{7}$ Life expectancy estimates for men and women in the United States fall far short of those in other industrialized nations, and yet the United States continues to represent a major outlier with respect to overall health-related spending. Therefore, the need to define and measure clinically relevant outcomes, quality, and value becomes ever greater as the relentless increase in expenditures continues unabated.

\section{Approaches to Value-Based Care}

Despite the practical challenges in quantifying quality and cost, the concept of value-based care ${ }^{10}$ continues to gain attention given concerns regarding whether modest survival gains justify significant cost increases and whether this represents a sustainable health care model. ${ }^{2}$ A better understanding of the value of cancer care is needed, as are strategies to improve its quality and value. However, the evaluation of health care delivery to patients with cancer is inherently complex. Any definition of value needs to encompass the entire landscape of care delivery and the resulting outcomes. These needs, in turn, are determined by the patient's clinical situation, involving an interrelated set of medical conditions that should be addressed in a coordinated and integrated fashion. As noted by Porter, 7 "value for the patient is created by providers' combined efforts over the full cycle of care. The benefits of any one intervention for ultimate outcomes will depend on the effectiveness of other interventions throughout the care cycle."

To effectively implement guidelines or policies to promote value-based care, the oncology community needs to arrive at a common definition of what value is in cancer care. Across all disciplines, value is recognized as a multidimensional concept that is complicated by the perspectives and goals of multiple parties. ${ }^{2}$ No universal consensus exists as to which stakeholders-patients, payers, physicians, policy makers, drug manufacturers, or societies - should have the dominant voice in assessing value. ${ }^{3}$ Although value clearly relates outcomes to costs, ${ }^{7}$ living longer also costs more, because patients who are alive will continue to incur medical costs, collect social security, and use resources. ${ }^{11,12}$ Given the complex nature and current poor performance of the US health care system, individual oncologists and their professional societies need to take an active major role in establishing a 
broad definition of value in oncology that can be used to guide value-based policies, and it must be one that can be understood by a range of stakeholders. ${ }^{13}$

Four parameters should be incorporated into the definition of value: survival, quality of care, cost, and equity. ${ }^{2,3,14}$ Survival is the most universally accepted and straightforward metric in cancer care. Quality of care is much more difficult to define and measure and usually refers to adherence to evidence-based guidelines or standard practice. Measurement of health care processes has become the dominant method with which to measure quality because of the inherent difficulties in measuring and comparing outcomes directly between varying patient populations. For example, in the Healthcare Effectiveness Data and Information Set measures, 73 of 78 are clear process measures, and none of the measures represent true outcomes. ${ }^{7}$ Despite this, efforts to systematically assess value using these metrics have been conducted. One notable example being the examination of head and neck cancer treatment at MD Anderson Cancer Center ${ }^{15}$ using the Porter ${ }^{7}$ model of value. In addition to the focus on providing high-quality care, efforts are underway to decrease practices in oncology that are believed to have little or no value, including overuse of antiemetic prophylaxis in low to moderate emetogenic chemotherapy, multidrug chemotherapy regimens in patients for whom single agents will suffice, routine surveillance PET scans, prostate-specific antigen screening in patients with an expected lifespan of less than 10 years, and the use of targeted therapies in patients in whom the therapeutic target has not been verified. ${ }^{16}$

Costs typically represent costs to the health care system or payer, but can also represent direct out-of-pocket costs, work lost, and indirect resource consumption, such as transportation, lodging, nonmedical supportive measures, and time spent by caretakers. Even direct costs, such as the cost of a specific drug, may vary substantially between institutions and patients, depending on negotiations between stakeholders. ${ }^{3}$ Equity represents the ability of all patients to receive the same level of care, but is typically more the exception than the rule because of inherent and poorly understood differences in patient access, cultural norms, and patient biology.

\section{Research Focus}

Given the inherent complexity of defining and measuring quality of care and costs in clinical oncology and how these in turn define value, comparative effectiveness research (CER) is needed to help with the discussion of value in cancer care. ${ }^{3,17}$ The goal of CER in oncology is to gather available evidence to address critical questions related to the benefits, harms, and overall value of interventions and to provide a framework with which to begin to address value-based care. Recommendations as to how oncology CER should be best conducted and presented in order to remain useful to practicing oncologists are now emerging, including the avoidance of technical jargon, outdated treatment modalities, inadequate sample sizes, unclear statistics, and ambiguous conclusions. ${ }^{17}$

One increasingly apparent principle of emerging therapeutics in oncology is that different patients and cancers exhibit highly variable responses to the same intervention. The potential for these highly variable responses are driving the need to establish clinical and molecular parameters to help assess an individual patient's likelihood of improvement for individual treatment options. The aggregation problem in health care refers to whether small benefits to many individuals should have priority over large benefits to only a few, as long as the aggregate benefit to the overall population is greater than the benefits to the few. ${ }^{1}$ The converse thinking can also be observed, wherein the impetus for administering expensive, generally non-cost-effective therapies is the potential for a significant and valuable benefit, albeit rare, at the individual patient level. Many people and patients support the use of interventions with the potential to provide large benefits to relatively few, especially when these interventions can be lifesaving, although the aggregate benefit to the population may be small. Which approach should have priority currently remains a sociopolitical as well as clinical debate, and will hopefully be aided in the future by more sophisticated personalization of care and more selective administration of interventions to those who stand to benefit most.

\section{Use Case: Colony-Stimulating Factors for the Prevention of Febrile Neutropenia}

Febrile neutropenia (FN) is a common and potentially serious and life-threatening complication of myelosuppressive cancer chemotherapy. Although a proportion of patients with $\mathrm{FN}$ are considered low 
Dinan et al

risk and may be safely treated in the ambulatory setting, prompt evaluation is essential with the collection of blood cultures and the empiric initiation of broad-spectrum antibiotics. The management of FN often necessitates hospitalization for this evaluation and urgent treatment. A small but clinically meaningful proportion of patients with $\mathrm{FN}$ experience prolonged, complicated hospitalizations, sometimes requiring admission to the intensive care unit, and are at considerable risk of early mortality. ${ }^{18}$ The use of prophylactic colony-stimulating factors (CSFs), administered after the first cycle of chemotherapy and before the development of neutropenia, can reduce the risk, severity, and duration of FN. The appropriate prophylactic use of myeloid growth factors has been shown in meta-analyses to reduce the relative risk of documented infections, ${ }^{19} \mathrm{FN},{ }^{20}$ and infection-related mortality, ${ }^{21}$ while allowing the maintenance or increase of chemotherapy dose-intensity. ${ }^{22}$ At the same time, considerable concern exists about the cost of these agents when used outside of current guideline recommendations.

\section{Current Guidelines}

Current guidelines from the EORTC, ${ }^{23} \mathrm{ASCO},{ }^{24}$ and $\mathrm{NCCN}^{25}$ uniformly recommend prophylactic administration of a CSF for chemotherapy regimens that pose a $20 \%$ or greater risk of $\mathrm{FN}$ based on published clinical trials. At the same time, they do not recommend routine prophylaxis with a CSF for regimens associated with less than a $10 \%$ risk of FN. Finally, the guidelines acknowledge a grey area for patients receiving chemotherapy associated with an intermediate risk of $\mathrm{FN}$ in the range of $10 \%$ to $20 \%$. For patients receiving myelosuppressive chemotherapy regimens with an intermediate risk of $\mathrm{FN}$, guidelines recommend assessing patient-specific risk factors. Although many such factors have been identified and risk models have been developed, the assessment of individual patient risk for $\mathrm{FN}$ remains an active area of investigation and implementation.

An analysis of randomized clinical trials of myelosuppressive chemotherapy regimens in nonHodgkin's lymphoma and early-stage breast cancer found that one-third or more of trials reported no hematologic toxicity data and even fewer studies reported data on chemotherapy dose-intensity, potentially limiting the generalizability of reported rates of treatment-related toxicity. ${ }^{26}$ Even when hematologic toxicity data were reported, methods of reporting were inconsistent and rates of published hematologic toxicities varied widely for the same or similar chemotherapy regimens. ${ }^{26}$ Overall, it is estimated that $25 \%$ to $40 \%$ of treatment-naïve patients develop FN during common chemotherapy regimens, ${ }^{27}$ although the risk varies widely depending on the patient population and dosage, timing, and type of chemotherapy used. The potential impact of supportive care measures, including the use of prophylactic antibiotics and myeloid growth factors, is also often poorly documented in clinical trials. At the same time, in actual practice, it has been suggested that CSFs are both underused for chemotherapy regimens associated with high risk of FN and overused for those associated with low risk. ${ }^{2}$

\section{Direct Costs of CSF Administration}

The major toxicity associated with CSF administration is bone pain. ${ }^{28}$ However, the key limiting factor preventing universal prophylactic administration of CSF in myelosuppressive regimens is often cost. The current cost of growth factor administration is substantial and represents a significant portion of cancer treatment-related health care costs, with total US sales of Neupogen and Neulasta in 2011 reaching $\$ 5.2$ billion. ${ }^{29}$ The direct cost of prophylactic CSF added to standard chemotherapy for non-Hodgkin's lymphoma in first-line treatment has been reported to be approximately $\$ 6000 .{ }^{30}$ Pegfilgrastim, a pegylated form of CSF that allows single-dose administration instead of multiple daily injections, has been found to be more cost-effective than daily filgrastim. ${ }^{31,32}$ It is anticipated that the cost of CSF administration will change in the near future with the introduction of biosimilars, also known as follow-on biologics. The introduction of tbo-filgrastim and forthcoming CSFs could drive down the price of the reference agents. ${ }^{33}$ However, whether biosimilars will be readily adopted into US clinical practice and how they will impact pricing and the relative cost-effectiveness of filgrastim and pegfilgrastim remain uncertain.

\section{Value and the CSFs}

Efforts to define the value of intervention with myeloid growth factors to reduce the risk and consequences of FN must consider the cost of the myeloid growth factor and the potential cost savings associated with preventing $\mathrm{FN}$ and its complications, including hospitalization, repeated visits to the emergency department of outpatient practice sites, and imaging and 
laboratory costs, as well as time lost from work for the patient and caregivers. In addition, when neutropenic complications reduce the ability to provide effective treatment, they may also increase the risk of disease recurrence, the cost of which needs to be considered, especially in patients treated with curative intent. Although myeloid growth factors are important contributors to the increasing costs of cancer care, their effectiveness at reducing the risk of life-threatening complications, costly hospitalizations, outpatient care, morbidity, and mortality also must be considered. Finding the balance between these clinical and economic outcomes is at the heart of attempting to define the value of an intervention such as the CSFs.

\section{Economic Analyses}

From a prevention perspective, recent economic analyses in the United States suggest that CSF prophylaxis becomes cost saving at a risk of FN of approximately $17 \%$ to $20 \%$, consistent with current guidelines based on results from the clinical studies. The primary cost savings associated with the use of CSF are attributed to the reduction of expensive inpatient hospitalizations, the costs of which have also increased over time. ${ }^{18,34}$ Early studies in the context of lower direct costs of hospitalization estimated cost savings only at higher risk of FN..$^{35,36}$ More recent median cost estimates on hospitalization for FN in patients with cancer have been in the range of $\$ 2000$ to $\$ 3000$ per day. Prevention of the frequency, intensity, or duration of hospitalization through the use of prophylactic CSF administration remains the primary means by which prophylaxis becomes costeffective or even cost-saving. ${ }^{32}$ In an economic analysis of women undergoing chemotherapy for ovarian cancer, the cost-effectiveness of prophylactic CSF was heavily dependent on the risk of FN. In low-risk patients with an estimated $5 \%$ risk of $\mathrm{FN}$, the cost of preventing a single hospitalization using primary prophylaxis was $\$ 47,000$. In contrast, prophylactic CSF administration in patients with a $16 \%$ risk of FN compared with secondary or therapeutic administration strategies was actually cost-saving. ${ }^{37}$ Similarly, a more recent economic analysis demonstrated that primary prophylaxis with pegfilgrastim was cost-effective compared with secondary prophylaxis for patients with recurrent ovarian cancer receiving docetaxel or topotecan. ${ }^{38}$

Prevention of even a few hospitalizations for FN can provide cost savings, with single admissions for
FN averaging $\$ 22,000$ or greater. ${ }^{39}$ Potential benefit in those receiving regimens with less than a 20\% risk of $\mathrm{FN}$ is supported by a recent Cochrane review, ${ }^{40}$ which found that in patients with breast cancer, prophylactic administration of CSFs was associated with reductions in $\mathrm{FN}$ events and need for hospital care in patients receiving chemotherapy with predominantly intermediate FN risk. In this analysis, only 2 of the 6 studies reported a rate of $\mathrm{FN}$ greater than $20 \%$, with most studies noting a risk between $10 \%$ and $20 \%$ and one study showing a risk of less than $10 \%$. Because of changes in supportive care, costs of CSF, and costs of inpatient care, the relative balance and cost-effectiveness of CSF prophylaxis represents a moving target and will likely require further evaluation over time. ${ }^{35}$

\section{Alternative Metrics}

Current recommendations for routine administration of prophylactic CSF for rates of FN greater than $20 \%$ are largely based on data from randomized trials of patients receiving higher-risk chemotherapy regimens. As noted previously, these recommendations have been supported by cost analyses considering the tradeoff between the costs of the myeloid growth factors and reductions in costs of hospitalization and accompanying care for FN. However, a more comprehensive consideration of value should encompass, in addition to cost, consideration of potential survival benefit, quality of life, and equity. The value of prophylactic CSF administration is not limited to the direct prevention of FN and subsequent complications, but also includes the enhanced capability of delivering full-dose chemotherapy on schedule for most patients. ${ }^{41} \mathrm{~A}$ large meta-analysis found that the prophylactic use of CSF across a variety of cancer settings increased the average dose density of chemotherapy by $8 \% .{ }^{21}$ When the perspectives of the patient and clinician are considered in the estimation of value, it is important to consider whether higher doses of chemotherapy and the ability to proceed with full dose-chemotherapy may result in improved disease control and possibly overall survival. ${ }^{41}$ Such an effect of CSF support on the administration of chemotherapy has been suggested by a recent meta-analysis of 60 randomized controlled trials of granulocyte CSF compared with controls across a range of cancer types and treatment regimens showing a significant reduction in all-cause mortality with a median follow-up of nearly 5 years. Notably, the 
magnitude of the survival impact increased with a longer duration of reported follow-up and with greater relative dose intensity across studies. ${ }^{23,41}$

\section{Conclusions}

A need exists for greater clarity and consistency in the definition and application of the relative value of diagnostic and therapeutic interventions in medical decision-making and health care delivery. Any consideration of value needs to encompass the broad landscape of health care delivery and clinical outcomes. Current guidelines recommend routine prophylactic administration of CSF in patients receiving chemotherapy associated with high risk of $\mathrm{FN}$, and recommend the assessment of patient-specific risk with intermediate- or low-risk chemotherapy regimens before considering CSF prophylaxis. Importantly, the guidelines explicitly exclude costs from consideration in the development of their recommendations. In fact, neither the regulatory approval of new interventions nor the development of clinical practice guidelines are explicitly informed by considerations of cost or overall value of strategies. ${ }^{12}$ At the same time, current guidelines do not consider all aspects of value in the care of patients with cancer, including the potential impact of neutropenic complications on patient quality of life and the potential effect of completing full-dose chemotherapy on time on subsequent disease control and survival, especially in the curative setting. ${ }^{23,25}$ Given the complexities inherent in evaluating quality of care, cost, and value in medical decision-making, oncologists need to take a major role in defining value in oncology and evaluating the relative or comparative value of diagnostic and therapeutic options available in order to guide better, clinically relevant, value-based policies. ${ }^{13}$

\section{References}

1. Schnipper LE, Meropol NJ, Brock DW. Value and cancer care: toward an equitable future. Clinical cancer research: an official journal of the American Association for Cancer Research 2010;16:6004-6008.

2. Ramsey S, Schickedanz A. How should we define value in cancer care? Oncologist 2010;15(Suppl 1):1-4.

3. Basch E, Prestrud AA, Hesketh PJ, et al. Antiemetics: American Society of Clinical Oncology clinical practice guideline update. J Clin Oncol 2011;29:4189-4198.

4. Garber A, Goldman DP, Jena AB. The promise of health care cost containment. Health Aff (Millwood) 2007;26:1545-1547.
5. Luengo-Fernandez R, Leal J, Gray A, Sullivan R. Economic burden of cancer across the European Union: a population-based cost analysis. Lancet Oncol 2013;14:1165-1174.

6. Keating NL, Landrum MB, Lamont EB, et al. Area-level variations in cancer care and outcomes. Med Care 2012;50:366-373.

7. Porter ME. What is value in health care? N Engl J Med 2010;363:24772481.

8. Hoverman J. Relating value in cancer care to cost and outcome. Oncology Business Review 2011;5:24-26.

9. Lyman GH. Counting the costs of cancer care. Lancet Oncol 2013;14:1142-1143.

10. Porter ME, Teisberg EO. How physicians can change the future of health care. JAMA 2007;297:1103-1111.

11. Liu L, Rettenmaier AJ, Saving TR. Comparative effectiveness and comparative costs. Oncology (Williston Park) 2010;24:537.

12. Outcomes of cancer treatment for technology assessment and cancer treatment guidelines. American Society of Clinical Oncology. J Clin Oncol 1996;14:671-679.

13. Lyman GH. Comparative effectiveness research in oncology. Oncologist 2013;18:752-759.

14. Konski A. Cost, quality, and value in healthcare: a new paradigm. Oncology (Williston Park) 2010;24:542-543.

15. Feeley TW, Fly HS, Albright $\mathrm{H}$, et al. A method for defining value in healthcare using cancer care as a model. Journal of healthcare management / American College of Healthcare Executives 2010;55:399-411.

16. Schnipper LE, Lyman GH, Blayney DW, et al. American Society of Clinical Oncology 2013 top five list in oncology. J Clin Oncol 2013;31:4362-4370.

17. Ramsey SD, Sullivan SD, Reed SD, et al. Oncology comparative effectiveness research: a multistakeholder perspective on principles for conduct and reporting. Oncologist 2013;18:760-767.

18. Kuderer NM, Dale DC, Crawford J, et al. Mortality, morbidity, and cost associated with febrile neutropenia in adult cancer patients. Cancer 2006;106:2258-2266.

19. Sung L, Nathan PC, Alibhai SM, et al. Meta-analysis: effect of prophylactic hematopoietic colony-stimulating factors on mortality and outcomes of infection. Ann Intern Med 2007;147:400-411.

20. Bohlius J, Reiser M, Schwarzer G, Engert A. Granulopoiesis-stimulating factors to prevent adverse effects in the treatment of malignant lymphoma. Cochrane Database Syst Rev 2004:CD003189.

21. Kuderer NM, Dale DC, Crawford J, Lyman GH. Impact of primary prophylaxis with granulocyte colony-stimulating factor on febrile neutropenia and mortality in adult cancer patients receiving chemotherapy: a systematic review. J Clin Oncol 2007;25:3158-3167.

22. Lyman GH, Kuderer NM. Epidemiology of febrile neutropenia. Support Cancer Ther 2003;1:23-35.

23. Aapro MS, Bohlius J, Cameron DA, et al. 2010 update of EORTC guidelines for the use of granulocyte-colony stimulating factor to reduce the incidence of chemotherapy-induced febrile neutropenia in adult patients with lymphoproliferative disorders and solid tumours. Eur J Cancer 2011;47:8-32.

24. Smith TJ, Khatcheressian J, Lyman GH, et al. 2006 update of recommendations for the use of white blood cell growth factors: an evidence-based clinical practice guideline. J Clin Oncol 2006;24:31873205.

25. Crawford J, Becker PS, Armitage J, et al. NCCN Clinical Practice Guidelines in Oncology: Myeloid Growth Factors. Version 2, 2014 Available at: NCCN.org. Accessed January 8, 2014.

26. Dale DC, McCarter GC, Crawford J, Lyman GH. Myelotoxicity and dose intensity of chemotherapy: reporting practices from randomized clinical trials. J Natl Compr Canc Netw 2003;1:440-454.

27. Dale DC. Colony-stimulating factors for the management of neutropenia in cancer patients. Drugs 2002;62(Suppl 1):1-15.

28. Filgrastim label information. Dailymed Web site. Available at: http:// dailymed.nlm.nih.gov/dailymed/lookup.cfm?setid=97cc73cc-b5b7-458aa933-77b00523e193. Accessed January 9, 2014.

29. Amgen 2011 Earnings Report. Availalbe at: http://www.amgen.com/media/ media_pr_detail.jsp?releaseID=1653300. Accessed January 24, 2014.

30. Doorduijn JK, Buijt I, van der Holt B, et al. Economic evaluation of prophylactic granulocyte colony stimulating factor during chemotherapy in elderly patients with aggressive non-Hodgkin's lymphoma. Haematologica 2004;89:1109-1117. 
Chemotherapy-Induced Neutropenia

31. Liu Z, Doan QV, Malin J, Leonard R. The economic value of primary prophylaxis using pegfilgrastim compared with filgrastim in patients with breast cancer in the UK. Appl Health Econ Health Policy 2009;7:193-205.

32. Eldar-Lissai A, Cosler LE, Culakova E, Lyman GH. Economic analysis of prophylactic pegfilgrastim in adult cancer patients receiving chemotherapy. Value Health 2008;11:172-179.

33. Hirsch BR, Lyman GH. Will biosimilars gain momentum? J Natl Compr Canc Netw 2013;11:1291-1297.

34. Lyman GH, Kuderer NM. The economics of the colony-stimulating factors in the prevention and treatment of febrile neutropenia. Crit Rev Oncol Hematol 2004;50:129-146.

35. Smith TJ. Economic analysis of the clinical uses of the colony-stimulating factors. Curr Opin Hematol 1996;3:175-179.

36. Lyman GH, Dale DC, Crawford J. Incidence and predictors of low doseintensity in adjuvant breast cancer chemotherapy: a nationwide study of community practices. J Clin Oncol 2003;21:4524-4531.
37. Numnum TM, Kimball KJ, Rocconi RP, et al. Pegfilgrastim for the prevention of febrile neutropenia in patients with epithelial ovarian carcinoma-a cost-effectiveness analysis. Int $\mathrm{J}$ Gynecol Cancer 2007;17:1019-1024.

38. Fust K, Li X, Maschio M, et al. Cost-effectiveness of prophylaxis treatment strategies for febrile neutropenia in patients with recurrent ovarian cancer. Gynecol Oncol 2014;133:446-453.

39. Michels SL, Barron RL, Reynolds MW, et al. Costs associated with febrile neutropenia in the US. Pharmacoeconomics 2012;30:809-823.

40. Renner P, Milazzo S, Liu JP, et al. Primary prophylactic colony-stimulating factors for the prevention of chemotherapy-induced febrile neutropenia in breast cancer patients. Cochrane Database Syst Rev 2012;10:CD007913.

41. Lyman GH, Dale DC, Culakova E, et al. The impact of the granulocyte colony-stimulating factor on chemotherapy dose intensity and cancer survival: a systematic review and meta-analysis of randomized controlled trials. Ann Oncol 2013;24:2475-2485. 